\title{
On bounds of the sine and cosine along straight lines on the complex plane
}

\author{
Feng Qi \\ College of Mathematics, \\ Inner Mongolia University for Nationalities, China \\ School of Mathematical Sciences, \\ Tianjin Polytechnic University, China \\ Institute of Mathematics, \\ Henan Polytechnic University, China \\ email: qifeng618@gmail.com; \\ qifeng618@hotmail.com; qifeng618@qq.com \\ url: https://qifeng618.wordpress.com
}

Abstract. In the paper, the author discusses and computes bounds of the sine and cosine along straight lines on the complex plane.

\section{Motivations}

In the theory of complex functions, the sine and cosine on the complex plane $\mathbb{C}$ are denoted and defined respectively by

$$
\sin z=\frac{e^{i z}-e^{-i z}}{2 i} \quad \text { and } \quad \cos z=\frac{e^{i z}+e^{-i z}}{2}
$$

where $z=x+i y, x, y \in \mathbb{R}$, and $i=\sqrt{-1}$ is the imaginary unit. When $z=x \in \mathbb{R}$, these two trigonometric functions become $\sin x$ and $\cos x$ which satisfy the periodicity

$$
\sin (x+2 k \pi)=\sin x, \quad \cos (x+2 k \pi)=\cos x
$$

2010 Mathematics Subject Classification: 33B10, 30A10

Key words and phrases: bound, sine, cosine, horizontal straight line, vertical straight line, sloped straight line, complex plane 
and the boundedness

$$
0 \leq|\sin x| \leq 1, \quad 0 \leq|\cos x| \leq 1
$$

for $k \in \mathbb{Z}$. On the other hand, when $z=i y$ for $y \in \mathbb{R}$,

$$
\sin (i y)=\frac{e^{-y}-e^{y}}{2 i} \rightarrow \pm i \infty \quad \text { and } \quad \cos (i y)=\frac{e^{-y}+e^{y}}{2} \rightarrow+\infty
$$

as $y \rightarrow \pm \infty$. These imply that the sine and cosine are bounded on the real $x$-axis, but unbounded on the imaginary $y$-axis.

Motivated by the above boundedness, we naturally guess that the complex functions $\sin z$ and $\cos z$ for $z \in \mathbb{C}$ are

1. bounded on all straight lines parallel to the real $x$-axis,

2. unbounded on all straight lines whose slopes are not horizontal.

In this paper, we will verify the above guesses and compute bounds for $\sin z$ and $\cos z$ on all horizontal straight lines.

\section{Unboundedness of sine and cosine on sloped and vertical lines}

On the sloped straight line $y=\alpha+\beta x$ for constants $\alpha \in \mathbb{R}$ and $\beta \neq 0$ on the complex plane $\mathbb{C}$, by the triangle inequality for complex numbers, we have

$$
\begin{aligned}
|\sin z| & =|\sin (x+i(\alpha+\beta x))| \\
& =\left|\frac{e^{i[x+i(\alpha+\beta x)]}-e^{-i[x+i(\alpha+\beta x)]}}{2 i}\right| \\
& =\left|\frac{e^{[i x-(\alpha+\beta x)]}-e^{-[i x-(\alpha+\beta x)]}}{2 i}\right| \\
& \geq \frac{1}{2}|| e^{[i x-(\alpha+\beta x)]}|-| e^{-[i x-(\alpha+\beta x)]}|| \\
& =\frac{1}{2}\left|e^{-(\alpha+\beta x)}-e^{(\alpha+\beta x)}\right| \\
& \rightarrow+\infty, \quad x \rightarrow \pm \infty
\end{aligned}
$$

and

$$
|\cos z|=|\cos (x+i(\alpha+\beta x))|
$$




$$
\begin{aligned}
& =\left|\frac{e^{i[x+i(\alpha+\beta x)]}+e^{-i[x+i(\alpha+\beta x)]}}{2}\right| \\
& =\left|\frac{e^{[i x-(\alpha+\beta x)]}+e^{-[i x-(\alpha+\beta x)]}}{2}\right| \\
& \geq \frac{1}{2}|| e^{[i x-(\alpha+\beta x)]}|-| e^{-[i x-(\alpha+\beta x)]}|| \\
& =\frac{1}{2}\left|e^{-(\alpha+\beta x)}-e^{(\alpha+\beta x)}\right| \\
& \rightarrow+\infty, \quad x \rightarrow \pm \infty .
\end{aligned}
$$

Consequently, the functions $\sin z$ and $\cos z$ are not bounded along any sloped straight line.

On the vertical straight line $x=\gamma$ for any constant $\gamma \in \mathbb{R}$ on the complex plane, by the triangle inequality for complex numbers, we have

$$
\begin{aligned}
|\sin z| & =|\sin (\gamma+i y)|=\left|\frac{e^{i(\gamma+i y)}-e^{-i(\gamma+i y)}}{2 i}\right| \\
& \geq \frac{1}{2}|| e^{i(\gamma+i y)}|-| e^{-i(\gamma+i y)}||=\frac{1}{2}\left|e^{-y}-e^{y}\right| \rightarrow+\infty
\end{aligned}
$$

and

$$
\begin{aligned}
|\cos z| & =|\cos (\gamma+i y)|=\left|\frac{e^{i(\gamma+i y)}+e^{-i(\gamma+i y)}}{2}\right| \\
& \geq \frac{1}{2}|| e^{i(\gamma+i y)}|-| e^{-i(\gamma+i y)}||=\frac{1}{2}\left|e^{-y}-e^{y}\right| \rightarrow+\infty
\end{aligned}
$$

as $y \rightarrow \pm \infty$. Consequently, the functions $\sin z$ and $\cos z$ are not bounded along any vertical straight line.

\section{Bounds of the sine on horizontal straight lines}

On the horizontal straight line $y=\alpha$ for any constant $\alpha \in \mathbb{R}$ on the complex plane $\mathbb{C}$, by the triangle inequality for complex numbers, we have

$$
\begin{aligned}
|\sin z| & =|\sin (x+i \alpha)|=\left|\frac{e^{i(x+i \alpha)}-e^{-i(x+i \alpha)}}{2 i}\right| \\
& =\left|\frac{e^{(i x-\alpha)}-e^{-(i x-\alpha)}}{2 i}\right|=\frac{1}{2}\left|\frac{e^{i x}}{e^{\alpha}}-e^{-i x} e^{\alpha}\right|
\end{aligned}
$$




$$
\leq \frac{1}{2}\left(\left|\frac{e^{i x}}{e^{\alpha}}\right|+\left|e^{-i x} e^{\alpha}\right|\right)=\frac{1}{2}\left(\frac{1}{e^{\alpha}}+e^{\alpha}\right)
$$

and

$$
\begin{aligned}
|\sin z| & =|\sin (x+i \alpha)|=\left|\frac{e^{i(x+i \alpha)}-e^{-i(x+i \alpha)}}{2 i}\right| \\
& =\left|\frac{e^{(i x-\alpha)}-e^{-(i x-\alpha)}}{2 i}\right|=\frac{1}{2}\left|\frac{e^{i x}}{e^{\alpha}}-e^{-i x} e^{\alpha}\right| \\
& \geq \frac{1}{2}|| \frac{e^{i x}}{e^{\alpha}}|-| e^{-i x} e^{\alpha}||=\frac{1}{2}\left|\frac{1}{e^{\alpha}}-e^{\alpha}\right| .
\end{aligned}
$$

Therefore, it follows that

$$
\frac{1}{2}\left|\frac{1}{e^{\alpha}}-e^{\alpha}\right| \leq|\sin (x+i \alpha)| \leq \frac{1}{2}\left(\frac{1}{e^{\alpha}}+e^{\alpha}\right), \quad x, \alpha \in \mathbb{R} .
$$

When $z=2 k \pi+i \alpha$ for $k \in \mathbb{Z}$, we have

$$
\sin z=\sin (2 k \pi+i \alpha)=\frac{e^{i(2 k \pi+i \alpha)}-e^{-i(2 k \pi+i \alpha)}}{2 i}=-\frac{i}{2}\left(\frac{1}{e^{\alpha}}-e^{\alpha}\right) .
$$

When $z=2 k \pi+\frac{\pi}{2}+i \alpha$ for $k \in \mathbb{Z}$, we have

$$
\begin{aligned}
\sin z & =\sin \left(2 k \pi+\frac{\pi}{2}+i \alpha\right)=\frac{e^{i(2 k \pi+\pi / 2+i \alpha)}-e^{-i(2 k \pi+\pi / 2+i \alpha)}}{2 i} \\
& =\frac{e^{i(\pi / 2+i \alpha)}-e^{-i(\pi / 2+i \alpha)}}{2 i}=\frac{e^{-\alpha}+e^{\alpha}}{2}=\frac{1}{2}\left(\frac{1}{e^{\alpha}}+e^{\alpha}\right) .
\end{aligned}
$$

When $z=(2 k+1) \pi+i \alpha$ for $k \in \mathbb{Z}$, we have

$$
\begin{aligned}
\sin z & =\sin ((2 k+1) \pi+i \alpha)=\frac{e^{i((2 k+1) \pi+i \alpha)}-e^{-i((2 k+1) \pi+i \alpha)}}{2 i} \\
& =\frac{e^{i(\pi+i \alpha)}-e^{-i(\pi+i \alpha)}}{2 i}=\frac{1}{2 i}\left(e^{\alpha}-\frac{1}{e^{\alpha}}\right)=-\frac{i}{2}\left(e^{\alpha}-\frac{1}{e^{\alpha}}\right) .
\end{aligned}
$$

When $z=(2 k+1) \pi+\frac{\pi}{2}+i \alpha$ for $k \in \mathbb{Z}$, we have

$$
\begin{aligned}
\sin z & =\sin \left((2 k+1) \pi+\frac{\pi}{2}+i \alpha\right) \\
& =\frac{e^{i((2 k+1) \pi+\pi / 2+i \alpha)}-e^{-i((2 k+1) \pi+\pi / 2+i \alpha)}}{2 i} \\
& =\frac{e^{i(3 \pi / 2+i \alpha)}-e^{-i(3 \pi / 2+i \alpha)}}{2 i}=-\frac{e^{-\alpha}+e^{\alpha}}{2}=-\frac{1}{2}\left(\frac{1}{e^{\alpha}}+e^{\alpha}\right) .
\end{aligned}
$$




\section{Bounds of the cosine on horizontal straight lines}

On the horizontal straight line $y=\alpha$ for any constant $\alpha \in \mathbb{R}$ on the complex plane $\mathbb{C}$, by the triangle inequality for complex numbers, we have

$$
\begin{aligned}
|\cos z| & =|\cos (x+i \alpha)|=\left|\frac{e^{i(x+i \alpha)}+e^{-i(x+i \alpha)}}{2}\right| \\
& =\left|\frac{e^{(i x-\alpha)}+e^{-(i x-\alpha)}}{2}\right|=\frac{1}{2}\left|\frac{e^{i x}}{e^{\alpha}}+e^{-i x} e^{\alpha}\right| \\
& \leq \frac{1}{2}\left(\left|\frac{e^{i x}}{e^{\alpha}}\right|+\left|e^{-i x} e^{\alpha}\right|\right)=\frac{1}{2}\left(\frac{1}{e^{\alpha}}+e^{\alpha}\right)
\end{aligned}
$$

and

$$
\begin{aligned}
|\cos z| & =|\cos (x+i \alpha)|=\left|\frac{e^{i(x+i \alpha)}+e^{-i(x+i \alpha)}}{2}\right| \\
& =\left|\frac{e^{(i x-\alpha)}+e^{-(i x-\alpha)}}{2}\right|=\frac{1}{2}\left|\frac{e^{i x}}{e^{\alpha}}+e^{-i x} e^{\alpha}\right| \\
& \geq \frac{1}{2}|| \frac{e^{i x}}{e^{\alpha}}|-| e^{-i x} e^{\alpha}||=\frac{1}{2}\left|\frac{1}{e^{\alpha}}-e^{\alpha}\right| .
\end{aligned}
$$

Therefore, it follows that

$$
\frac{1}{2}\left|\frac{1}{e^{\alpha}}-e^{\alpha}\right| \leq|\cos (x+i \alpha)| \leq \frac{1}{2}\left(\frac{1}{e^{\alpha}}+e^{\alpha}\right), \quad x, \alpha \in \mathbb{R} .
$$

When $z=2 k \pi+i \alpha$ for $k \in \mathbb{Z}$, we have

$$
\cos z=\cos (2 k \pi+i \alpha)=\frac{e^{i(2 k \pi+i \alpha)}+e^{-i(2 k \pi+i \alpha)}}{2}=\frac{1}{2}\left(\frac{1}{e^{\alpha}}+e^{\alpha}\right) .
$$

When $z=2 k \pi+\frac{\pi}{2}+i \alpha$ for $k \in \mathbb{Z}$, we have

$$
\begin{aligned}
\cos z & =\cos \left(2 k \pi+\frac{\pi}{2}+i \alpha\right)=\frac{e^{i(2 k \pi+\pi / 2+i \alpha)}+e^{-i(2 k \pi+\pi / 2+i \alpha)}}{2} \\
& =\frac{e^{i(\pi / 2+i \alpha)}+e^{-i(\pi / 2+i \alpha)}}{2}=\frac{i e^{-\alpha}-i e^{\alpha}}{2}=\frac{i}{2}\left(\frac{1}{e^{\alpha}}-e^{\alpha}\right) .
\end{aligned}
$$

When $z=(2 k+1) \pi+i \alpha$ for $k \in \mathbb{Z}$, we have

$$
\cos z=\cos ((2 k+1) \pi+i \alpha)=\frac{e^{i((2 k+1) \pi+i \alpha)}+e^{-i((2 k+1) \pi+i \alpha)}}{2}
$$




$$
=\frac{e^{i(\pi+i \alpha)}+e^{-i(\pi+i \alpha)}}{2}=-\frac{1}{2}\left(\frac{1}{e^{\alpha}}+e^{\alpha}\right) .
$$

When $z=(2 k+1) \pi+\frac{\pi}{2}+i \alpha$ for $k \in \mathbb{Z}$, we have

$$
\begin{aligned}
\cos z & =\cos \left((2 k+1) \pi+\frac{\pi}{2}+i \alpha\right) \\
& =\frac{e^{i((2 k+1) \pi+\pi / 2+i \alpha)}+e^{-i((2 k+1) \pi+\pi / 2+i \alpha)}}{2} \\
& =\frac{e^{i(3 \pi / 2+i \alpha)}+e^{-i(3 \pi / 2+i \alpha)}}{2}=\frac{-i e^{-\alpha}+i e^{\alpha}}{2}=-\frac{i}{2}\left(\frac{1}{e^{\alpha}}-e^{\alpha}\right) .
\end{aligned}
$$

\section{$5 \quad$ Alternative proofs}

Since $\sin \left(z+\frac{\pi}{2}\right)=\cos z$ for $z \in \mathbb{C}$, there is a similar behaviour of sine and cosine in the complex plane $\mathbb{C}$. Hence, in what follows, we just only consider sine.

It is easy to see that sine, cosine, hyperbolic sine, and hyperbolic cosine have relations

$$
\sin (i t)=i \sinh t, \quad \sinh (i t)=i \sin t, \quad \cos (i t)=\cosh t, \quad \cosh (i t)=\cos t .
$$

Accordingly, we have

$$
\sin z=\sin (x+i y)=\sin x \cos (i y)+\cos x \sin (i y)=\sin x \cosh y+i \cos x \sinh y
$$

and

$$
|\sin z|^{2}=\sin ^{2} x \cosh ^{2} y+\cos ^{2} x \sinh ^{2} y .
$$

On any horizontal line $y=c$, say, we have

$$
\begin{aligned}
|\sin z|^{2} & =\sin ^{2} x \cosh ^{2} c+\cos ^{2} x \sinh ^{2} c \\
& =\sin ^{2} x \cosh ^{2} c+\cos ^{2} x\left(\cosh ^{2} c-1\right)=\cosh ^{2} c-\cos ^{2} x
\end{aligned}
$$

or

$$
|\sin z|^{2}=1+\sinh ^{2} c-\cos ^{2} x=\sinh ^{2} c+\sin ^{2} x .
$$

Consequently, sine is bounded on all horizontal lines.

Look at a non-horizontal line, where $z=\gamma+\alpha x+i \beta y$ for $\beta \neq 0$ (by nonhorizontality). Here

$$
|\sin z|^{2}=\sin ^{2}(\gamma+\alpha x) \cosh ^{2}(\beta y)+\cos ^{2}(\gamma+\alpha x) \sinh ^{2}(\beta y) .
$$


If the line is sloped so that $\alpha \neq 0$ (by non-verticality), then both terms in the above equation are unbounded, so that sine is unbounded.

If the line is vertical, so that $\alpha=0$, we have to be a tad careful! If $\gamma$ is not a multiple of $\pi$, the term $\sin ^{2}(\gamma+\alpha x) \cosh ^{2}(\beta y)$ is unbounded; and if $\gamma$ is a multiple of $\pi$, then the term $\cos ^{2}(\gamma+\alpha x) \sinh ^{2}(\beta y)$ is unbounded. In a word, sine is unbounded on all non-horizontal lines.

\section{Conclusions}

On the sloped straight line $y=\alpha+\beta x$ for $\alpha \in \mathbb{R}$ and $\beta \neq 0$ on the complex plane $\mathbb{C}$, the trigonometric functions $\sin z=\sin (x+\mathfrak{i}(\alpha+\beta x))$ and $\cos z=$ $\cos (x+i(\alpha+\beta x))$ are unbounded.

On the vertical straight line $x=\gamma$ for any scalar $\gamma \in \mathbb{R}$ on the complex plane $\mathbb{C}$, the trigonometric functions $\sin z=\sin (\gamma+i y)$ and $\cos z=\cos (\gamma+i y)$ are unbounded.

On the horizontal straight line $y=\alpha$ for any constant $\alpha \in \mathbb{R}$ on the complex plane $\mathbb{C}$, the trigonometric functions $\sin z=\sin (x+i \alpha)$ and $\cos z=\cos (x+i \alpha)$ are bounded by the double inequalities

$$
|\sinh \alpha| \leq|\sin (x+i \alpha)| \leq \cosh \alpha, \quad x, \alpha \in \mathbb{R}
$$

and

$$
|\sinh \alpha| \leq|\cos (x+i \alpha)| \leq \cosh \alpha, \quad x, \alpha \in \mathbb{R}
$$

whose equalities are respectively attained at points

$$
2 k \pi+i \alpha, \quad 2 k \pi+\frac{\pi}{2}+i \alpha, \quad(2 k+1) \pi+i \alpha, \quad 2 k \pi+\frac{3 \pi}{2}+i \alpha
$$

with concrete values

$$
\begin{aligned}
& \sin (2 k \pi+i \alpha)=\cos \left(2 k \pi+\frac{3 \pi}{2}+i \alpha\right)=i \sinh \alpha, \\
& \sin \left(2 k \pi+\frac{\pi}{2}+i \alpha\right)=\cos (2 k \pi+i \alpha)=\cosh \alpha, \\
& \sin ((2 k+1) \pi+i \alpha)=\cos \left(2 k \pi+\frac{\pi}{2}+i \alpha\right)=-i \sinh \alpha, \\
& \sin \left(2 k \pi+\frac{3 \pi}{2}+i \alpha\right)=\cos ((2 k+1) \pi+i \alpha)=-\cosh \alpha
\end{aligned}
$$

for $k \in \mathbb{Z}$. 
Letting $\alpha \rightarrow 0$ in the double inequalities (2) and (3) recovers inequalities in (1) for $x \in \mathbb{R}$.

On the horizontal belt zones $0 \leq A \leq y \leq B$ and $-B \leq y \leq-A \leq 0$ on the complex plane $\mathbb{C}$, the trigonometric functions $\sin z=\sin (x+i y)$ and $\cos z=\cos (x+i y)$ are bounded by the double inequalities

$$
\sinh A \leq|\cos (x \pm i y)| \leq \cosh B
$$

and

$$
\sinh A \leq|\sin (x \pm i y)| \leq \cosh B
$$

for $x \in \mathbb{R}$.

\section{$7 \quad$ An open problem}

The inequalities in (1) can be refined as

$$
\frac{2}{\pi} x \leq \sin x \leq x \text { and } 1-\frac{2}{\pi} x \leq \cos x \leq 1-\frac{x^{2}}{\pi}
$$

for $0 \leq x \leq \frac{\pi}{2}$. See [1, p. 143], [3, p. 22], and [4, p. 33]. These two double inequalities in (4) are respectively called as Jordan's and Kober's inequality. These two double inequalities have been further refined, generalized, applied, and surveyed in the papers $[2,5,6,8,9,10]$ and closely related references therein. Motivated by these refinements, generalizations, and applications, we pose an open problem: can one refine, generalize, and apply the double inequalities (2) and (3) for $x \in\left[0, \frac{\pi}{2}\right]$ and $\alpha \neq 0$ ?

Finally we remark that this paper is a revised version of the preprint [7].

\section{Acknowledgements}

The author is grateful to anonymous referees for their careful corrections to and valuable comments on the original version of this paper.

\section{References}

[1] P. S. Bullen, A Dictionary of Inequalities, Pitman Monographs and Surveys in Pure and Applied Mathematics 97, Addison Wesley Longman Limited, 1998. 
[2] Z.-H. Huo, D.-W. Niu, J. Cao, and F. Qi, A generalization of Jordan's inequality and an application, Hacet. J. Math. Stat., 40 (2011), no. 1, $53-61$.

[3] H. Kober, Approximation by integral functions in the complex domain, Trans. Amer. Math. Soc., 56 (1944), no. 1, 7-31; Availble online at https: //doi.org/10.2307/1990275.

[4] D. S. Mitrinović, Analytic Inequalities, In cooperation with P. M. Vasić. Die Grundlehren der mathematischen Wissenschaften, Band 165 Springer-Verlag, New York-Berlin, 1970.

[5] D.-W. Niu, J. Cao, and F. Qi, Generalizations of Jordan's inequality and concerned relations, Politehn. Univ. Bucharest Sci. Bull. Ser. A Appl. Math. Phys., 72 (2010), no. 3, 85-98.

[6] D.-W. Niu, Z.-H. Huo, J. Cao, and F. Qi, A general refinement of Jordan's inequality and a refinement of $L$. Yang's inequality, Integral Transforms Spec. Funct. 19 (2008), no. 3, 157-164; Available online at http://dx. doi.org/10.1080/10652460701635886.

[7] F. Qi, On bounds of the sine and cosine along straight lines on the complex plane, Preprints 2018, 2018090365, 5 pages; Available online at https: //doi.org/10.20944/preprints201809.0365.v1.

[8] F. Qi and B.-N. Guo, A criterion to justify a holomorphic function, Glob. J. Math. Anal., 5 (2017), no. 1, 24-26; Available online at https://doi. org/10.14419/gjma.v5i1.7398.

[9] F. Qi and Q.-D. Hao, Refinements and sharpenings of Jordan's and Kober's inequality, Mathematics and Informatics Quarterly, 8 (1998), no. $3,116-120$.

[10] F. Qi, D.-W. Niu, and B.-N. Guo, Refinements, generalizations, and applications of Jordan's inequality and related problems, J. Inequal. Appl., 2009 (2009), Article ID 271923, 52 pages; Available online at http://dx.doi.org/10.1155/2009/271923. 